\title{
A CUT-OFF PRIORITY FLOW CLASSIFICA- TION POLICY FOR DATA-DRIVEN IP/ATM SWITCHING SYSTEMS
}

\author{
Jun Zheng and Victor O. K. Li, Fellow, IEEE \\ Department of Electrical and Electronic Engineering \\ The University of Hong Kong \\ Pokfulam Road, Hong Kong \\ Email: (jzheng,vli\}@eee.hku.hk
}

\begin{abstract}
Flow classification is one of the key issues in the design of a data-driven IP/ATM switching system. To classify flows by applications is an effective approach in a real system due to its simplicity in implementation. In this paper, we propose a cut-off priority flow classification policy to improve the application-based flow classification policy. The basic idea is to assign priorities to the applications that are selected for ATM switching so that those comparatively longer-lived applications may have better performance under heavy traffic load. Performance analysis is also carried out to show the efficiency of this cut-off priority flow classification policy.
\end{abstract}

Keywords: Flow Classification, Cut-off Priority, IP, ATM Switching.

\section{INTRODUCTION}

Flow classification is one of the key issues in the design of a data-driven IP/ATM switching system [1]. To perform flow classification, a data-driven IP/ATM switching system must have a local flow classification policy/algorithm to decide whether or not an IP flow should be selected for ATM switching. Different policies may have different impacts on the system performance. For this reason, alternative flow classification policies have been proposed for better performance, including the protocol-based policy, the application-based policy, the $\mathrm{X} / \mathrm{Y}$ algorithm [2] and the adaptive algorithm [3]. In this paper, we study flow classification policies and propose a cut-off priority policy to improve the application-based policy. The basic idea is to assign priorities to the applications that are 
selected for ATM switching. Higher priorities are assigned to those comparatively longer-lived applications so that they may have better performance under heavy traffic load. Performance analysis based on queuing theory is also carried out to show the efficiency of this cut-off priority flow classification policy.

The remainder of this paper is organised as follows. In Section 2, we give a brief review of flow classification policies. In Section 3, we present our proposed cut-off priority flow classification policy and analyse its performance. In Section 4, we give some numerical results to show its performance as compared with that of the application-based policy. In Section 5, we conclude our study.

\section{FLOW CLASSIFICATION}

The main function of flow classification in a data-driven IP/ATM switching system is to identify flows and decide whether they should be switched directly in the ATM hardware or forwarded by the routing software. This can be implemented by inspecting the values of the packet header fields and making a decision based upon a local policy. For this purpose, a datadriven IP/ATM switching system must have a flow classification policy/algorithm. Different policies may result in different system performance. For this reason, alternative flow classification policies have been proposed for better performance. Generally, long-lived flows with a large number of packets should be selected for ATM switching while shortlived flows with a small number of packets should be handled by normal hop-by-hop forwarding [1]. The designer may choose to implement a particular policy according to the traffic characteristics in the specific environment the system operates in.

One flow classification policy, the protocol-based policy [2], is to classify flows by protocols. With this policy, all TCP flows are selected for ATM switching while all UDP flows are forwarded by the routing software. The argument is that connection-oriented services generally last longer and have more packets to be sent over a short time than connectionless services. Similarly, flows can also be classified by applications (or port numbers), such as ftp (20), telnet (23) and http (80). This application-based policy [2] is dependent on the statistical measurement of the mean duration and the average number of packets of each flow. Only those applications that tend to generate long-lived flows and contain a large number of packets are selected for ATM switching. Both of the above two policies have the advantage of simplicity in implementation. 
Another flow classification policy [4] is to count the number of packets received on each flow so that the first $\mathrm{X}$ packets of each flow are forwarded by the routing software while the subsequent packets are switched by the ATM hardware, independent of protocols and applications. A modified version of this policy is the $\mathrm{X} / \mathrm{Y}$ policy [2]. This policy also counts the number of packets received on each flow. If the number of packets of a flow received within $\mathrm{Y}$ seconds exceeds $\mathrm{X}$, further packets of the flow will be switched by the ATM hardware. The basis behind these two policies is that if $\mathrm{X}$ packets have already been received on a flow (within $\mathrm{Y}$ seconds), it is reasenable to expect that there will be more packets on this flow. The simulation studies in [2] and [4] showed that these two policies could separate short-lived flows from long-lived flows more effectively as compared with the protocol-based and the application-based policies.

All the above flow classification policies are static in that their criteria and control parameters (like $\mathrm{X}$ and $\mathrm{Y}$ ) are statically set. However, due to the varying characteristics of IP traffic, it is difficult to predict the traffic characteristics exactly and set optimal criteria or control parameters. For this reason, a static flow classification policy that works well in one system and/or for one application today may perform poorly in other systems and/or for other applications in the future. To adapt to the varying traffic characteristics, an adaptive flow classification algorithm has been proposed in [3]. This adaptive algorithm dynamically adjusts the values of control parameters in response to the varying traffic load and tries to balance the utilization of the system resources. The simulation study showed that it could offer better performance than the static policies. However, the cost of such an adaptive algorithm in terms of the complexity in implementation and the additional load on the processor might be much higher than that of the static policies.

\section{CUT-OFF PRIORITY POLICY}

Even though a variety of flow classification policies have been proposed, to classify flows by applications is still an effective approach in a real datadriven IP/ATM switching system due to its simplicity in implementation, especially when real-time multimedia applications are introduced. In such a switching system, when a flow is selected for ATM switching, the system will request a virtual circuit identifier (VCI) for the flow from its VC space so that the flow can be switched directly in the ATM hardware. However, due to the limited VC space of a real system, a flow that is selected for ATM switching might be blocked at the VC space and thus could not be switched 
in the ATM hardware. From the viewpoint of resource utilization, the VC space should be used as fully as possible in order to have better performance. In this sense, it is desirable to select as many applications as possible for ATM switching. On the other hand, if too many applications are selected for ATM switching indiscriminately, those applications that last comparatively longer and contain a larger number of packets might be blocked at the VC space under heavy traffic load, which may affect the system performance. To take account of both the VC utilization and the system performance, we now propose a flow classification policy based on cut-off priority.

\subsection{Policy Description}

The proposed cut-off priority policy is based on the application-based policy, i.e. it classifies flows by applications. Normally, a set of applications is selected for ATM switching. These applications are assigned a certain number of priorities according to the statistical measurement of their mean duration and average number of packets. Higher priorities are assigned to those applications that last comparatively longer and contain a larger number of packets. Correspondingly, a certain number of thresholds are set for the usage of the VC space. In general, we assume $k$ priorities and set $(k-1)$ thresholds for the usage of the VC space. When the VC usage exceeds threshold $i(i=1,2, \cdots, k-1)$, the flows with priority $j(1 \leq j \leq i)$ will no longer be selected for ATM switching. Only the flows with priority $j$ $(i<j \leq k)$ will be switched in the ATM hardware. For example, when the VC usage exceeds threshold 3 , the flows with priority 1, priority 2 and priority 3 will not be selected for ATM switching. Only the flows with priority 4 , priority $5, \ldots$, priority $k$ will be switched. It is conceivable that, with this cut-off priority policy, the number of higher priority flows switched can be efficiently increased under heavy traffic load as compared with that using the application-based policy. Meanwhile, the VC utilization will not decrease under light traffic load.

\subsection{Performance Analysis}

While there are many performance metrics for a data-driven IP/ATM switching system, we use the average number of packets switched in the system as the main metric in the following analysis. To analyse the performance, we use the performance model we proposed in [5], shown Figure 1. For comparison, we also discuss the performance of the application-based policy without cut-off priority. 


\subsubsection{Cut-Off Priority Policy}

As mentioned earlier, we assume $k$ priorities and $(k-1)$ thresholds. The probability that a flow is selected for ATM switching and it belongs to priority $i$ is $P_{i}$ and the average number of packets in a single priority-i flow is $L_{i}(i=1,2, \cdots, k)$, which can be estimated from empirical data. The threshold values are $C_{i}(i=1,2, \cdots, k-1)$, where $0 \leq C_{1} \leq C_{2} \leq \cdots C_{k-1} \leq C$ and $C$ is the VC space size. According to the performance model, for the cut-off priority policy, the VC space of the system can be modelled as an $M / M / C / C$ loss system with $k$ flow priorities. The arrival rate of priority-i flows to the VC space is $\lambda_{i}=P_{i} \lambda$, where $\lambda$ is the total flow arrival rate to the system. The service rate for priority-i flows is $\mu_{i}$, which can also be estimated from measurements.

$M / M / \infty$

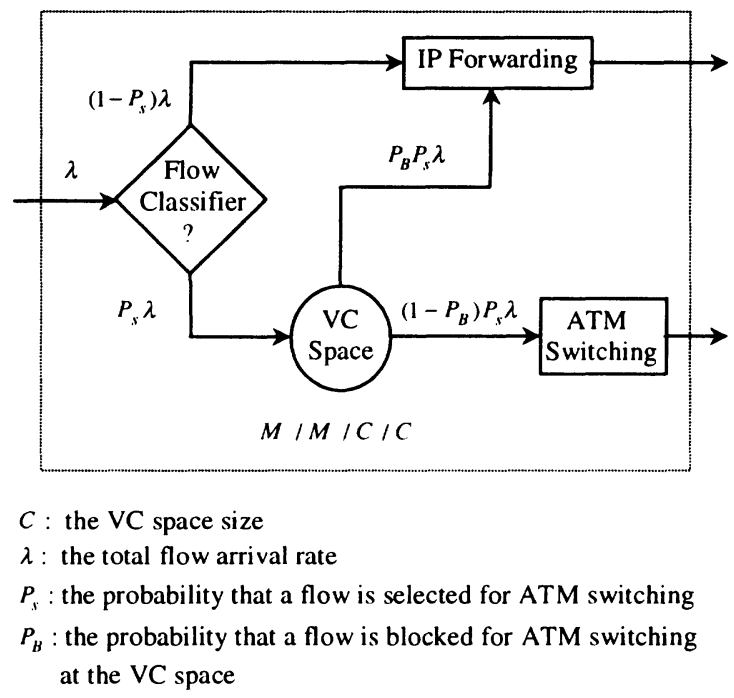

Figure 1 Performance Model

To perform the analysis, we use $n$ to describe the state that is defined by

$$
\bar{n}=\left(n_{1}, n_{2}, \cdots, n_{k}\right)
$$

and $\Omega$ to describe a set of allowable states that is defined by

$$
\Omega=\left\{\bar{n}: \quad 0 \leq n_{i} \leq C_{i}, \quad i=1,2, \cdots k ; \quad 0 \leq \sum_{i=1}^{k} n_{i} \leq C\right\}
$$

where $n_{i}$ is the number of priority-i flows using the VC. Another notation needed is $n_{i}^{+}$that is defined by 


$$
n_{i}^{+}=\left(n_{1}, \cdots, n_{i-1}, n_{i}+1, n_{i+1}, \cdots, n_{k}\right)
$$

Then we can use the results obtained in [6]. The joint steady state distribution is given by

where

$$
\begin{gathered}
P(\bar{n})=\prod_{i=1}^{k} \frac{a_{i}^{n_{i}}}{n_{i} !} \cdot G^{-1}(\Omega) \\
G(\Omega)=\sum_{n \in \Omega}\left(\prod_{i=1}^{k} \frac{a_{i}^{n_{i}}}{n_{i} !}\right)
\end{gathered}
$$

$a_{i}=\lambda_{i} / \mu_{i}$ is the offered load of priority-i flows and $1 / \mu_{i}$ is the mean duration of priority-i flows. The blocking probability experienced by a priority-i flow is

$$
P_{i B}=\frac{G\left(B_{i}^{+}\right)}{G(\Omega)}
$$

where $B_{i}^{+}$is defined by $\quad B_{i}^{+}=\left\{\bar{n} \in \Omega: n_{i}^{+} \notin \Omega\right\}$.

The steady state distribution is given by

$$
P\left(n_{i}\right)=\sum_{\substack{n \in \Omega \\ n_{i} \equiv n_{i}}} P(\bar{n}) \quad 0 \leq n_{i} \leq C_{i}
$$

Therefore, the average number of priority-i flows switched is

$$
N_{i}=\sum_{n_{i}=0}^{C_{i}} n_{i} P\left(n_{i}\right) \quad 0 \leq i \leq k
$$

and the total average number of packets switched can be calculated as

$$
N_{p c t}=\sum_{i=1}^{k} L_{i} N_{i}
$$

\subsubsection{Application-Based Policy}

For the application-based policy, the VC space is completely shared by all priority flows. Compared with the analysis for the cut-off priority policy, the set of allowable states under the same conditions now becomes

$$
\Omega=\left\{\bar{n}: \quad 0 \leq n_{i} \leq C, \quad i=1,2, \cdots k ; \quad 0 \leq \sum_{i=1}^{k} n_{i} \leq C\right\}
$$

All other analytical steps are the same as that for the cut-off priority policy. 


\section{NUMERICAL RESULTS}

In this section, we give some numerical results to illustrate the performance of the cut-off priority policy as compared with that of the application-based policy. For simplicity, we only discuss the case of $k=2$. For this purpose, we assume that $P_{1}=0.5, P_{2}=0.3, L_{1}=1000$ packets, $L_{2}=5000$ packets, $C=100, \mu_{1}=0.2$ flows $/ \mathrm{min}, \mu_{2}=1$ flow $/ \mathrm{min}$.

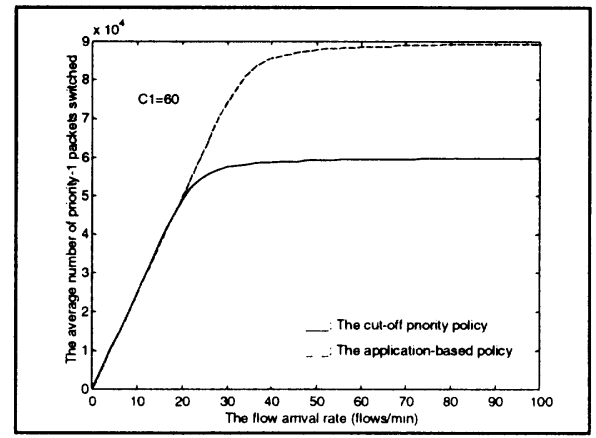

Figure 2 Avg. number of priority-1 packets switched versus the flow arrival rate

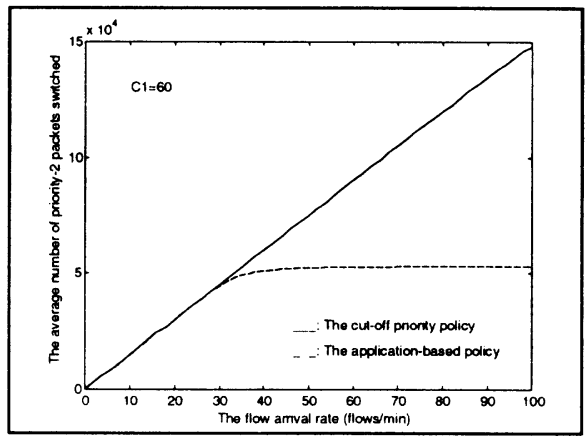

Figure 3 Avg. number of priority-2 packets switched versus the flow arrival rate

Figure 2 and Figure 3 respectively illustrate the comparison in the average number of priority-1 packets switched and the average number of priority-2 packets switched under a given threshold value $\left(C_{1}=60\right)$. When the flow arrival rate is very small, there is almost no difference in the performance for the two policies. This is because the VC usage will not reach the threshold under light traffic load. With the flow arrival rate increasing, the average number of priority-2 packets switched for the cut-off priority policy increases quickly while that for the application-based policy almost does not increase. On the contrary, the average number of priority-1 packets switched for the application-based policy is much larger than that for the cut-off priority policy. This is because, when the flow arrival rate is beyond a certain value, the VC usage will exceed the threshold. In this case, no more priority-1 flows will be selected for ATM switching, and only priority-2 flows will be switched. Therefore, with the cut-off priority policy, the performance of priority-2 applications in terms of the average number of packets switched is increased at the expense of decreased performance of priority-1 applications. However, this does not mean that the system performance in terms of the total average number of packets switched is also increased. 


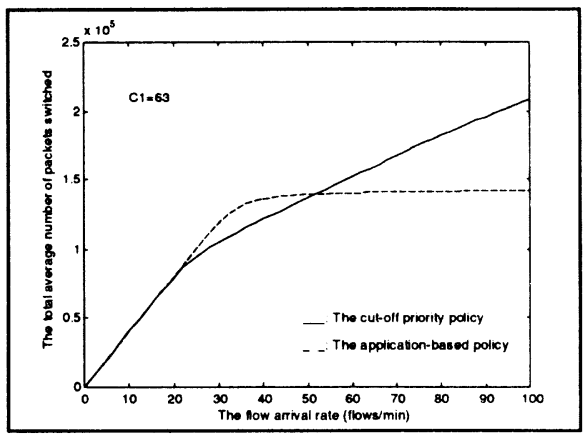

Figure 4 The total average number of packets switched versus the flow arrival rate

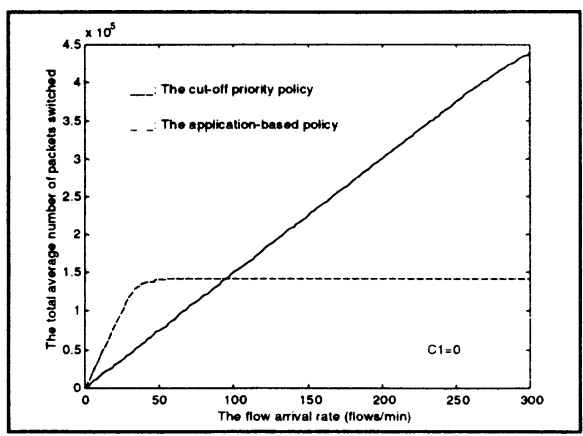

Figure 6 The total average number of packets switched versus the flow arrival rate

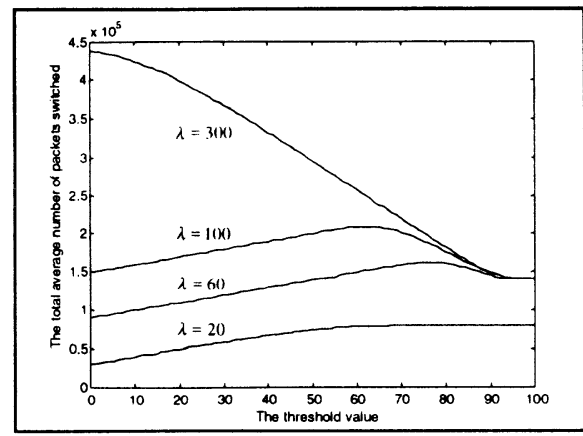

Figure 5 The total average number of packets switched versus the threshold value

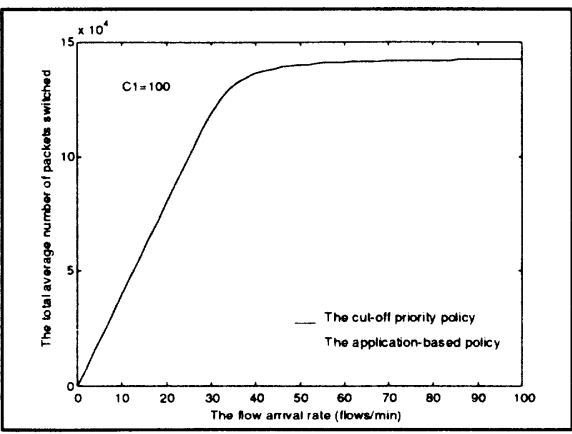

Figure 7 The total average number of packets switched versus the flow arrival rate

Figure 4 illustrates the comparison in the total average number of packets switched under a given threshold value $\left(C_{1}=63\right)$. It can be seen that the total average number of packets switched for the cut-off priority policy is not always larger than that for the application-based policy under different flow arrival rates. When the flow arrival rate is very small, there is almost no difference in the performance. With the flow arrival rate increasing, the performance for the application-based policy is better than that for the cut-off priority policy within a certain range of the flow arrival rate. Only when the flow arrival rate increases beyond a certain value will the performance for the cut-off priority policy become better than that for the application-based policy. The higher the flow arrival rate, the better the performance. Does this mean that, for some flow arrival rates, the total average number of packets switched for the cut-off priority policy is always smaller than that for the application-based policy? 
To answer this question, let us see Figure 5, which illustrates the total average number of packets switched for the cut-off priority policy versus the threshold value under different flow arrival rates. For a given flow arrival rate, the threshold value may be adjusted to have better performance. No matter how large the flow arrival rate is, an optimal threshold value may be found that produces the best performance. Different flow arrival rates may have different optimal threshold values. For example, when the flow arrival rate is 60 flows $/ \mathrm{min}$ and 100 flows/min, the optimal threshold value is 78 and 63 respectively. When the flow arrival rate is $20 \mathrm{flows} / \mathrm{min}$, the optimal threshold value is 100 , which is the VC space size. This means that, when the flow arrival rate is very small, the VC space is large enough to accommodate all the traffic. Accordingly, there is no need to set the threshold. When the flow arrival rate is 300 flows $/ \mathrm{min}$, the optimal threshold value is 0 , which is another extreme. In this case, the flow arrival rate is very high. To provide better performance for priority-2 applications, the whole VC space should be dedicated to priority-2 applications.

Figure 4 and Figure 6 show the comparison in the total number of packets switched between the two policies under the optimal threshold value $\left(C_{1}=63\right)$ for 100 flows $/ m i n$ and the optimal threshold value $\left(C_{1}=0\right)$ for 300 flows/min respectively. Obviously, the performance for the cut-off priority policy is better than that for the application-based policy in both cases. The higher the flow arrival rate, the smaller the optimal threshold value and the better the performance. In Figure 7, the threshold value is 100, which corresponds to the optimal threshold value for 20 flows $/ \mathrm{min}$. Accordingly, no threshold is actually set and the performance for the cut-off priority policy is neither improved nor decreased.

Basically, given the VC space size and a flow arrival rate, the performance for the cut-off priority policy in terms of the total average number of packets switched under the corresponding optimal threshold value can be efficiently improved as compared with that for the application-based policy. The higher the flow arrival rate, the better the performance. The only exception occurs when the flow arrival rate is very small, in which case the performance is unchanged.

Based on the above observations, a further refinement of this cut-off priority policy is to adjust the thresholds to match the varying IP traffic. For this purpose, the system periodically measures the average flow arrival rate, calculates the corresponding optimal threshold values and then dynamically adjusts the threshold values. 


\section{CONCLUSION}

In this paper, we proposed a cut-off priority flow classification policy for a data-driven IP/ATM switching system. The numerical results showed that, with the cut-off priority policy, the system performance in terms of the average number of higher-priority packets switched can be efficiently improved under heavy traffic load as compared with that using the application-based policy. Meanwhile, the optimal performance in terms of the total average number of packets switched in the system can be achieved by using the optimal threshold value of a given flow arrival rate. Even though the numerical analysis is discussed in the two-priority case, the results obtained apply to multiple priorities. Furthermore, by measuring the flow arrival rate and dynamically adjusting the threshold values, this cut-off priority flow classification policy will match the varying characteristic of IP traffic.

\section{Acknowledgement}

The authors would like to thank Dr. G. L. Li for his kind assistance in providing the valuable reference.

\section{References}

[1] Bruce Davie, Paul Doolan and Yakov Rekhter, "Switching in IP Networks," Morgan Kaufmann Publishers, 1998.

[2] Steven Lin and Nick Mckeown, "A Simulation Study of IP Switching," ACM SIGCOMM' 97, pp.15-24.

[3] Hao Che, San-qi Li and Arthur Lin, "Adaptive Resource Management for Flow-Based IP/ATM Hybrid Switching Systems," IEEE/ACM Transactions on Networking, Vol. 6, No. 5, Oct. 1998, pp. 544-557.

[4] Peter Newman, Greg Minshall and Thomas L. Lyon, "IP SwitchingATM under IP," IEEE/ACM Transactions on Networking, Vol. 6, No. 2, April 1998, pp. 117-129.

[5] Jun Zheng and Victor O. K. Li, "Performance Model for IP Switch," IEE Electronics Letters, Vol. 34, No. 21, Oct. 1998, pp. 2010-2011.

[6] Joseph S. Kaufman, "Blocking in a Shared Resource Environment," IEEE Transactions on Communications, Vol. Com-29, No. 10, Oct. 1981, pp. 1474-1481. 